\title{
Effect of ARID1A/BAF250a expression on carcinogenesis and clinicopathological factors in pure-type clear cell adenocarcinoma of the ovary
}

\author{
MASAFUMI KATO $^{1}$, MASASHI TAKANO ${ }^{1}$, MORIKAZU MIYAMOTO $^{1}$, NAOKI SASAKI ${ }^{1}$, TOMOKO GOTO ${ }^{1}$, \\ AYAKO SUZUKI $^{1}$, JUNKO HIRATA ${ }^{1}$, HIDENORI SASA ${ }^{1}$, HITOSHI TSUDA ${ }^{2}$ and KENICHI FURUYA ${ }^{1}$ \\ Departments of ${ }^{1}$ Obstetrics and Gynecology, and ${ }^{2}$ Basic Pathology, \\ National Defense Medical College Hospital, Tokorozawa, Saitama 359-8513, Japan
}

Received March 9, 2016; Accepted July 21, 2016

DOI: $10.3892 / \mathrm{mco} .2016 .973$

\begin{abstract}
Frequent mutation of the ARIDIA gene has been recently identified in ovarian clear-cell adenocarcinoma (CCA); however, the clinical significance of BAF250a expression encoded by the ARIDIA gene remains to be determined. The aim of the present study was to assess whether BAF250a expression had an impact on the clinical features of CCA. A total of 97 cases of CCA treated at a single institution were enrolled in the present study. The tissue samples were evaluated by immunohistochemical staining. BAF250a-deficient expression was observed in $30 \%$ (29/97) of all CCA cases. Of this, $19 \%$ of non-atypical endometriosis, $26 \%$ of atypical endometriosis, $39 \%$ of endometriosis-related CCA, $5 \%$ of benign clear-cell adenofibroma (CCAF), 5\% of borderline CCAF and $10 \%$ of CCAF-related CCA. BAF250a-deficient expression was significantly more frequent in endometriosis-related CCA compared with that in CCAF-related CCA $(\mathrm{P}=0.02)$. No significant difference was observed in the response rate of primary chemotherapy according to BAF250a expression status ( $\mathrm{P}=0.48)$. Additionally, BAF250a expression status was not significantly correlated with progression-free and overall survival in patients with CCA. Although loss of BAF250a expression was associated with early tumorigenesis in endometriosis-related CCA, this alteration was not significantly correlated with chemosensitivity and prognoses of CCA. Further biomarker analyses, including BAF250a expression, are required to improve the prognoses of CCA.
\end{abstract}

Correspondence to: Dr Masashi Takano, Department of Obstetrics and Gynecology, National Defense Medical College Hospital, 3-2 Namiki, Tokorozawa, Saitama 359-8513, Japan E-mail: mastkn@ndmc.ac.jp

Key words: ovarian cancer, clear cell adenocarcinoma, ARIDIA, BAF250a, endometriosis, progression-free survival, overall survival

\section{Introduction}

Ovarian clear-cell adenocarcinoma (CCA) has been recognized as a distinct entity among epithelial ovarian carcinomas (EOCs) with respect to its chemoresistant nature and frequent concurrence of endometriosis and clear-cell adenofibroma (CCAF). In Western populations, CCAs account for $~ 5-13 \%$ of all EOCs, whereas in Japan, its prevalence rises to $15-25 \%$ of all EOCs (1-3). Approximately two thirds of CCAs were diagnosed at early-stage disease; however, CCA has a relatively resistant phenotype to platinum-based chemotherapy, resulting in extremely poor prognosis, irrespective of surgical stages (4). Therefore, it is essential to elucidate a novel therapeutic target for CCA and to develop novel therapeutic strategies.

High-grade serous ovarian cancer, which is a major subtype of all histological subtypes, is characterized by TP53 mutations and frequent mutations or defects in BRCA1/2 pathway. By contrast, CCA appears to harbor a different molecular profile, including activating mutations in $P I K 3 C A$, and loss of PTEN and ARIDIA (5-9). ARIDIA mutation, in particular, is frequently observed in endometriosis-associated ovarian clear-cell and endometrioid adenocarcinoma, and it has been suggested that the mutation is as an early molecular event in the development of endometriosis-related CCA $(8,9,10)$. These distinct molecular features of CCA serve emphasis on the requirement to develop subtype-specific therapeutic approachs in the management of EOC.

Additionally, previous reports have suggested that CCAs are classified into two distinct molecular subtypes and that these subtypes have different clinical outcome (11). It was demonstrated that endometriosis-related CCA and CCAF-related CCA had different carcinogenic pathways $(12,13)$. Certain previous reports suggested that ARIDIA somatic mutation and subsequent BAF250a protein loss in CCA was correlated with response to chemotherapy and poor prognosis (14); however, other previous reports revealed no significance $(10,15-17)$. To date, the impact of BAF250a protein expression in response to primary chemotherapy and the prognoses of CCAs has remains to be determined.

The aim of the present study was to clarify whether loss of BAF250a expression correlated with early tumorigenesis of 
$\mathrm{CCA}$, and to evaluate the significance of BAF250a-deficient expression on clinicopathological variables in CCAs in a large series of patients treated at a single institution.

\section{Materials and methods}

Patients. A total of 97 cases of CCA treated between 1984 and 2007 at the National Defense Medical Coll ege Hospital, (Tokorozawa, Japan) were enrolled in the present study. Of the $97 \mathrm{CCAs}$, a consecutive series of $38 \mathrm{CCAs}$ synchronous with endometriosis (EM-related CCAs) and 21 CCAs adjacent to CCAF component (CCAF-related CCAs) were identified, according to the histopathological criteria described previously (18). Of those, 31 non-atypical endometrioses, 38 atypical endometrioses, 20 benign CCAFs and 21 borderline CCAFs were identified. A total of 18 cases with solitary endometriosis that had no CCA were used as controls. All patients provided written informed consent for the present study.

Immunohistochemical (IHC) staining. Two core specimens, $1.5 \mathrm{~mm}$ in diameter, for each case were obtained from cancer tissue blocks and transferred to recipient blocks using a Tissue Microarrayer (Beecher Instrument, Silver Spring, MD, USA). All specimens were cut into $4-\mu \mathrm{m}$-thick slices to make tissue sections for IHC staining. The tissue sections were deparaffinized and boiled in an autoclave at $121^{\circ} \mathrm{C}$ for $15 \mathrm{~min}$ in $0.01 \mathrm{~mol} / \mathrm{l}$ citrate buffer (pH 6.0) and were then allowed to cool at room temperature. Endogenous peroxidase activity was blocked using methanol added to $0.3 \%$ hydrogen peroxidase. The slides were incubated at $4^{\circ} \mathrm{C}$ overnight with mouse monoclonal primary antibody against BAF250a (cat. no. sc-32761; dilution, 1:100; Santa Cruz Biotechnology, Inc., Santa Cruz, CA, USA). Following incubation, the samples were reacted with dextran polymer reagent combined with secondary antibodies and peroxidase (cat. no. Z0420; 1:200; Dako A/S, Glostrup, Denmark) for $1 \mathrm{~h}$ at room temperature. Specific antigen-antibody reactions were visualized with $0.2 \%$ diaminobenzidine tetrahydrochrolide and hydrogen peroxidase, and counterstaining was performed using Mayer's hematoxylin. Non-neoplastic cells, including fibroblasts and lymphocytes, served as positive internal controls. As negative controls, tissue sections without the primary antibody were used.

For BAF250a detection, the presence of nuclear immunoreaction was taken into account for assignment of immuno-positivity. The lesions were considered to be positive for BAF250a if 50\% or more of tumor cells in the area of interest showed equal to or more strong immunoreactive intensity compared with the positive controls (BAF250a-retained cases). If no detectable nuclear staining of tumor cells or $<50 \%$ of tumor cells in the area of interest showed less immunoreactive intensity compared with the positive controls, they were defined as having a loss of BAF250a expression (BAF250a-deficient cases). The lesions were assessed independently by two observers (Masafumi Kato and Morikazu Miyamoto) in a blinded manner and any discrepancies between the two observers were resolved by conferring over a multi-viewer microscope.
Patient characteristics. Patient background, including age, concurrence of endometriosis, co-existence of CCAF, international federation of gynecology and obstetrics (FIGO) stage, residual tumor in primary surgery and chemotherapy regimen were compared, according to the BAF250a expression status. In addition, frequencies of loss of BAF250a expression in EM-related CCAs and CCAF-related CCAs were examined, as well as precursors (non-atypical endometrioses, atypical endometrioses, benign CCAFs and borderline CCAFs). Tumor response to adjuvant chemotherapy in evaluable cases, progression-free survival and overall survival were analyzed according to BAF250a expression status. Multivariate analyses for overall survival and progression-free survival were performed.

Statistical analysis. Statistical analyses were performed using Stat Mate IV software (ATMS, Tokyo, Japan) and Statview version 5 software (SAS Institute Japan, Ltd., Tokyo, Japan). Student's t-test and $\chi^{2}$ test were used to compare patient characteristics of two groups. For survival analyses, Kaplan-Meier curves and the log rank test were used. Prognostic significance was analyzed using the Cox proportional hazard model using variables as follows: Age (continuous variable), concurrence of endometriosis (yes vs. no), FIGO stage (I/II vs. III/IV), residual tumor ( $\leq 1 \mathrm{~cm}$ vs. $>1 \mathrm{~cm}$ ) and BAF250a status (BAF250a-deficient vs. BAF250a-retained). P $<0.05$ was considered to indicate a statistically significant difference.

\section{Results}

Association between patient characteristics and expression of BAF250a. The characteristics of the patients were assessed according to the BAF250a expression status, and this is shown in Table I. BAF250a-deficient expression was observed in 30\% (29/97) of all cases. No differences were observed in age, FIGO stage, residual tumor in primary surgery and chemotherapy regimen between the two groups. Concurrence of endometriosis was observed more frequently in BAF250a-deficient cases compared with in BAF250a-retained cases $(\mathrm{P}<0.05)$. By contrast, co-existence of CCAF was significantly more frequent in BAF250a-retained cases compared with that in BAF250a-deficient cases $(\mathrm{P}=0.04)$.

Frequency of BAF250a expression. The frequencies of BAF250a-deficient expression were 19\% (6/31) in non-atypical endometriosis, 26\% (10/38) in atypical endometriosis, 5\% $(1 / 20)$ in benign CCAF, $5 \%(1 / 21)$ in borderline CCAF, 39\% $(15 / 38)$ in EM-related CCA and 10\% (2/21) in CCAF-related CCA (Fig. 1). In solitary endometriosis, loss of BAF250a expression was detected in 6\% (1/18) of cases. In comparison with the frequency of BAF250a-deficient expression between EM-related CCAs and CCAF-related CCAs, a significant difference was observed between the two groups $(\mathrm{P}=0.02)$.

Response rate of chemotherapy. The response rate of chemotherapy in evaluable cases is shown in Table II. No significant difference was observed in response rate of primary chemotherapy between the two groups. A total of 50\% (13/26) in BAF250a-retained cases and 30\% (3/10) in BAF250a-deficient cases $(\mathrm{P}=0.48)$ was observed. 
Table I. Patient characteristics according to the expression of BAF250a.

\begin{tabular}{|c|c|c|c|}
\hline Characteristic & BAF250a-deficient cases $(n=29)$ & BAF250a-retained cases $(n=68)$ & P-value \\
\hline Median age (range) & $51(36-67)$ & $52(35-75)$ & 0.09 \\
\hline Concurrence of endometriosis & & & $<0.05$ \\
\hline Yes & $20(69 \%)$ & $32(47 \%)$ & \\
\hline No & $9(31 \%)$ & $36(53 \%)$ & \\
\hline Co-existence of CCAF & & & 0.04 \\
\hline Yes & $2(7 \%)$ & $19(28 \%)$ & \\
\hline No & $27(93 \%)$ & $49(72 \%)$ & \\
\hline FIGO stage & & & 0.17 \\
\hline Stage I/II & $15(52 \%)$ & $45(66 \%)$ & \\
\hline Stage III/IV & $14(48 \%)$ & $23(34 \%)$ & \\
\hline Residual tumor in primary surgery & & & 0.14 \\
\hline $0 \mathrm{~cm}$ & $15(52 \%)$ & $42(62 \%)$ & \\
\hline$\leqq 1 \mathrm{~cm}$ & $4(14 \%)$ & $12(18 \%)$ & \\
\hline$>1 \mathrm{~cm}$ & $10(34 \%)$ & $14(21 \%)$ & \\
\hline Chemotherapy regimen & & & 0.85 \\
\hline Cyclophosphamide + adriamycin + cisplatin & $12(41 \%)$ & $27(40 \%)$ & \\
\hline Irinotecan + cisplatin & $8(28 \%)$ & $24(35 \%)$ & \\
\hline Paclitaxel + carboplatin/cisplatin & $3(10 \%)$ & $7(10 \%)$ & \\
\hline None/unknown & $6(21 \%)$ & $10(15 \%)$ & \\
\hline
\end{tabular}

CCAF, clear-cell adenofibroma; FIGO, international federation of gynecology and obstetrics.

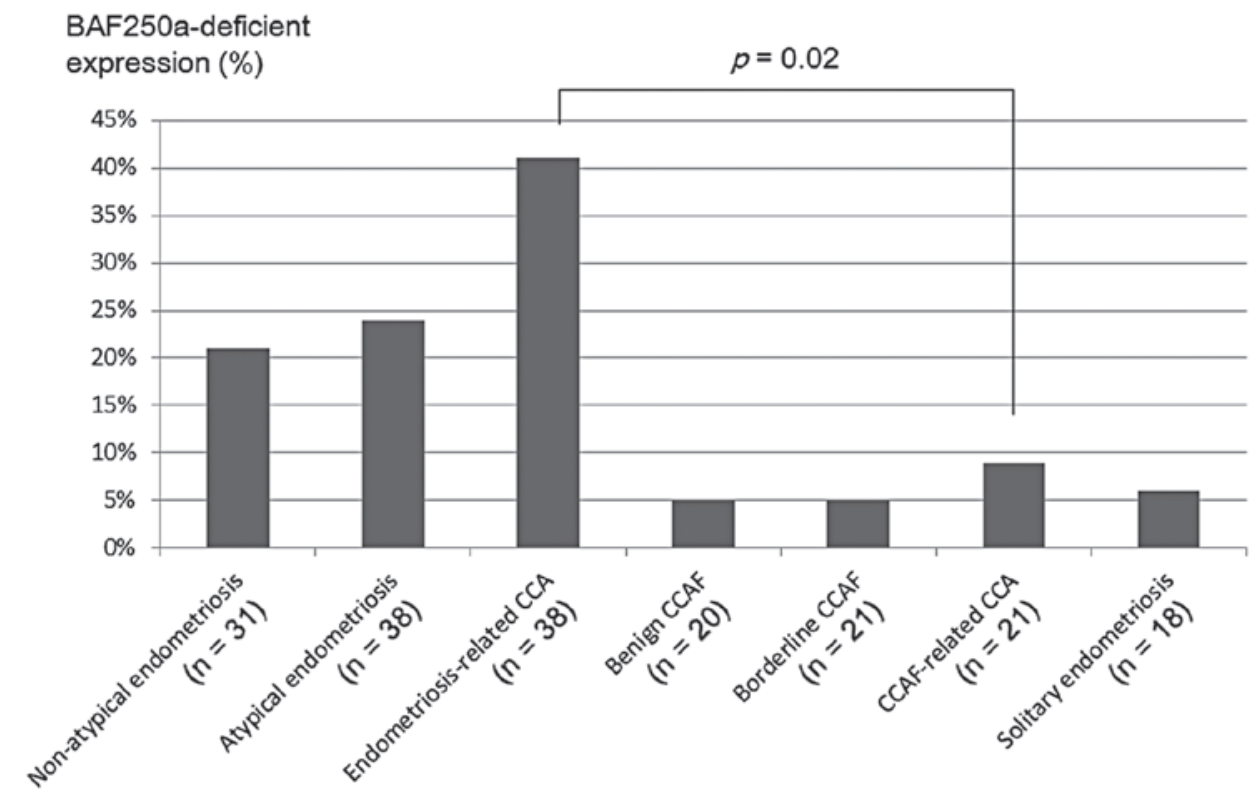

Figure 1. Frequencies of BAF250a-deficient expression in CCA and its precursors. Frequencies of BAF250a-deficient expression were 19\% (6/31) in non-atypical EM, 26\% (10/38) in atypical EM, 39\% (15/38) in EM-related CCA, 1/20 (5\%) in benign clear cell adenofibroma (CCAF), 1/21 (5\%) in borderline CCAF and 2/21 (10\%) in CCAF-related CCA. In solitary EM (control), the frequency of BAF250a-deficient expression was 6\% (1/18). BAF250a-deficient expression in EM-related CCA and its precursor was more frequently observed compared with in CCAF-related CCA and its precursor. BAF250a-deficient expression was significantly more frequent in EM-related CCA compared with in CCAF-related CCAF ( $\mathrm{P}=0.015)$. CCA, clear cell adenocarcinoma; EM, endometrioses; CCAF, clear-cell adenofibroma.

Overall and progression-free survival of the patients. Kaplan-Meier survival curves of all patients are shown in Fig. 2. BAF250a-deficient expression status was not signifi- cantly correlated with progression-free and overall survival of CCA in all enrolled cases. The 5-year progression-free survival rates were $68 \%$ in BAF250a-retained cases and $59 \%$ 
Table II. Tumor response of primary chemotherapy in evaluable cases with ovarian clear cell adenocarcinoma.

\begin{tabular}{lcc}
\hline RECIST assessment & BAF250a-deficient cases $(\mathrm{n}=10)$ & BAF250a-retained cases $(\mathrm{n}=26)$ \\
\hline Complete response & $2(20 \%)$ & $8(31 \%)$ \\
Partial response & $1(10 \%)$ & $5(19 \%)$ \\
Stable disease & $1(10 \%)$ & $3(16 \%)$ \\
Progressive disease & $6(60 \%)$ & $10(38 \%)$ \\
Response rate & $3 / 10(30 \%)$ & $13 / 26(50 \%)$
\end{tabular}

Response rate $=$ complete response + partial response for all patients. RECIST, response evaluation criteria in solid tumor.

A Cumulative survival rate

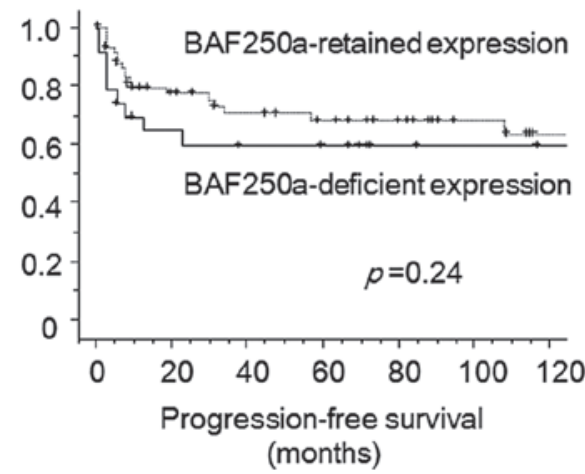

B Cumulative survival rate

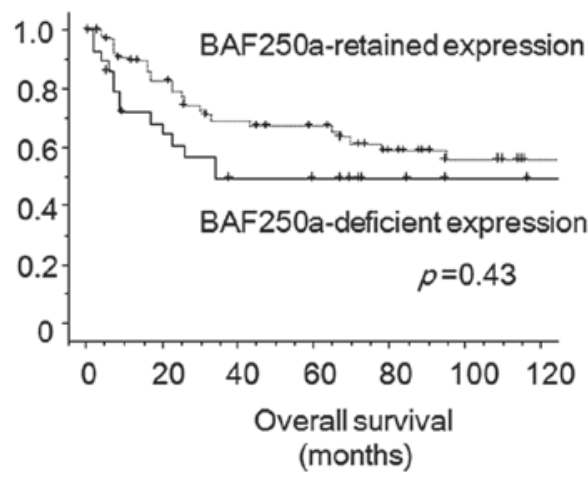

Figure 2. Kaplan-Meier survival curves of all enrolled cases according to the expression status of BAF250a. Progression-free and overall survival curves of BAF250a-deficient expression cases (solid line) and BAF250a-retained cases (dotted line) were produced. (A) The 5-year progression-free survival rate was 59\% in BAF250a-deficient expression cases and $68 \%$ in BAF150a-retained cases $(\mathrm{P}=0.24)$. (B) The 5-year overall survival rate was $49 \%$ in BAF250a-deficient expression cases and 67\% in BAF150a-retained cases $(\mathrm{P}=0.43)$.

in BAF250a-deficient cases $(\mathrm{P}=0.24)$. Additionally, the 5-year overall survival rates were $67 \%$ in retained expression cases and $49 \%$ in deficient cases $(\mathrm{P}=0.43)$.

Kaplan-Meier survival curves of stage I/II patients (Fig. 3A and B) and stage III/IV patients (Fig. 3C and D), according to BAF250a expression, were also shown. Additionally, no significant differences were observed in the progression-free and overall survival in patients with stage I/II cases. The 5-year progression-free survival was $86 \%$ in BAF250a-retained cases and $91 \%$ in BAF250a-deficient cases $(\mathrm{P}=0.82)$. The 5 -year overall survival rate was $85 \%$ in BAF250a-retained cases and 78\% in BAF250a-deficient cases $(\mathrm{P}=0.38)$. In cases with stage III/IV disease, no differences were observed between the two groups. The 5-year progression-free survival was $23 \%$ in BAF250a-retained cases and $13 \%$ in BAF250a-deficient cases $(\mathrm{P}=0.23)$. The 5-year overall survival rate was $25 \%$ in BAF250a-retained cases and $16 \%$ in BAF250a-deficient cases $(\mathrm{P}=0.21)$.

BAF250a expression status is not an independent prognostic factor. In the multivariate analysis for progression-free survival, BAF250a expression status was not identified as an independent prognostic factor ( $\mathrm{P}=0.47$; Table III). Residual tumor diameter was identified as an independent factor for progression-free survival. In the multivariate analysis for overall survival, age $(\mathrm{P}=0.01)$, FIGO stage $(\mathrm{P}<0.01)$ and residual tumor diameter $(\mathrm{P}=0.02)$ were prognostic factors; however, the BAF250a expression status was not identified as an independent prognostic factor $(\mathrm{P}=0.56)$ in the present cases.

\section{Discussion}

Frequencies of BAF250a-deficient expression in the present study were $6 \%$ in solitary endometriosis, $19 \%$ in non-atypical endometriosis, $26 \%$ in atypical endometriosis, $5 \%$ in benign CCAF, $5 \%$ in borderline CCAF and $30 \%$ in CCA. Previous reports have documented that frequencies of BAF250a-deficient expression in solitary endometriosis, atypical endometriosis and CCA were 0-15, 38.5 and 15-66\%, respectively $(10,14,19-21)$. When compared with solitary endometriosis and non-atypical endometriosis synchronous with CCA, BAF250a-deficient expression was more frequent in non-atypical endometriosis (6 vs. 19\%), suggesting this alteration was an early molecular alteration in the development of CCA. In addition, BAF250a-deficient expression in EM-related CCA and its precursor were more frequent compared with that in CCAF-related CCA and its precursor. These findings suggested that EM-related CCA may have different carcinogenic pathway from CCAF-related CCA.

Systematic review of BAF250a in response to the primary chemotherapy and prognosis in CCA was shown in Table IV. It remains controversial whether BAF250a status is correlated with the chemoresistance of CCA. A report demonstrated that loss of BAF250a expression was associated with 
Table III. Multivariate analyses for progression-free survival and overall survival.

\begin{tabular}{|c|c|c|c|c|c|c|}
\hline \multirow[b]{2}{*}{ Characteristic } & \multicolumn{3}{|c|}{ Progression-free survival } & \multicolumn{3}{|c|}{ Overall survival } \\
\hline & HR & $95 \% \mathrm{CI}$ & P-value & HR & $95 \% \mathrm{CI}$ & P-value \\
\hline Age (continuous variable) & 0.97 & $0.92-1.02$ & 0.30 & 0.94 & $0.90-0.99$ & 0.01 \\
\hline $\begin{array}{l}\text { Concurrence of endometriosis } \\
\text { (yes vs. no) }\end{array}$ & 0.78 & $0.32-1.86$ & 0.57 & 0.96 & $0.47-1.94$ & 0.90 \\
\hline FIGO stage (III/IV vs. I/II) & 9.10 & $3.33-25.0$ & $<0.01$ & 6.17 & $2.60-14.6$ & $<0.01$ \\
\hline Residual tumor $(>1 \mathrm{~cm}$ vs. $\leqq 1 \mathrm{~cm})$ & 1.79 & $0.68-4.72$ & 0.23 & 2.72 & $1.19-6.23$ & 0.02 \\
\hline $\begin{array}{l}\text { BAF250a expression } \\
\text { (retained vs. deficient) }\end{array}$ & 0.72 & $0.32-1.74$ & 0.47 & 0.80 & $0.39-1.67$ & 0.56 \\
\hline
\end{tabular}

HR, hazard ratio; CI, confidence interval; FIGO, international federation of gynecology and obstetrics.

A Cumulative survival rate

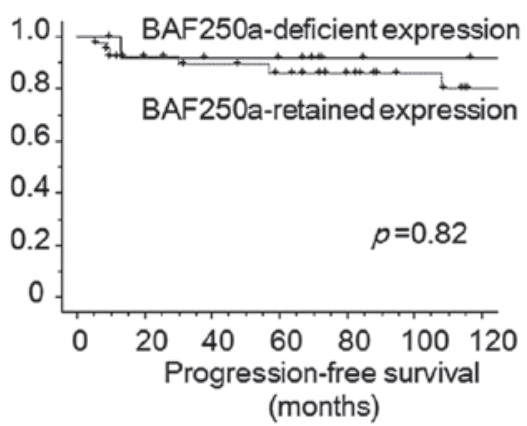

C

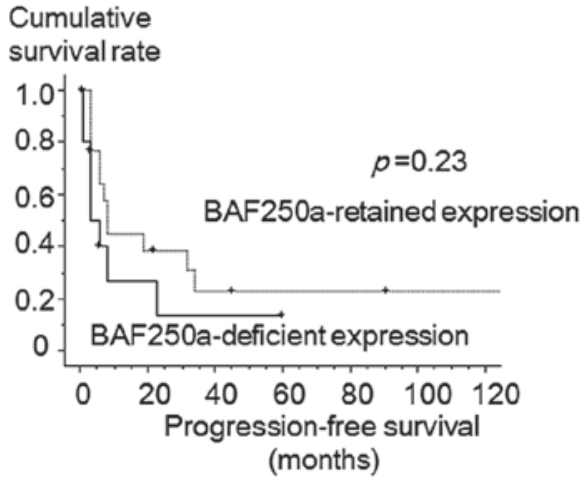

B Cumulative survival rate

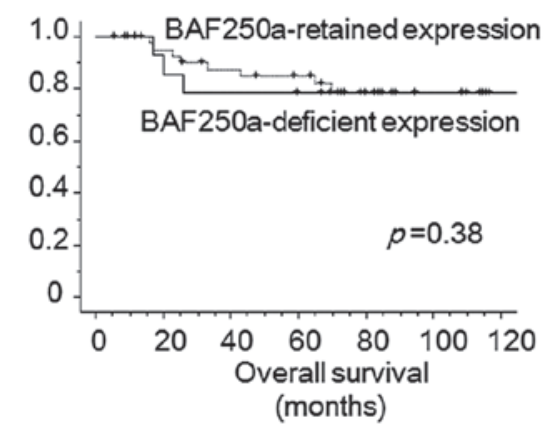

D

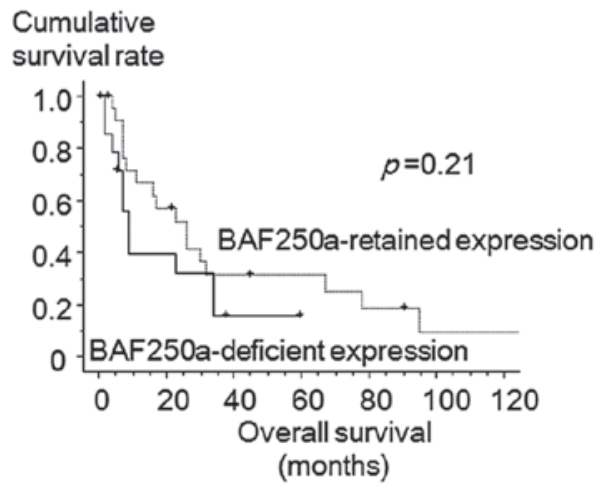

Figure 3. Kaplan-Meier survival curves according to the expression status of BAF250a. (A) Progression-free survival curves of patients with stage I/II disease. The 5-year progression-free survival rate was $91 \%$ in BAF250a-deficient cases and $86 \%$ in $\mathrm{BAF} 250 \mathrm{a}-\mathrm{retained}$ expression cases $(\mathrm{P}=0.82)$. $(\mathrm{B}) \mathrm{The}$ overall survival curves of the patients with stage I/II disease were produced. The 5 -year overall survival rate was $78 \%$ in BAF 250 a-deficient cases and $85 \%$ in BAF250a-retained expression cases $(\mathrm{P}=0.38)$. (C) The progression-free survival curves of patients with stage III/IV disease were plotted. The 5-year progression-free survival rate was $13 \%$ in BAF250a-deficient cases and 23\% in BAF250a-retained cases (P=0.23). (D) The overall survival curves of stage III/IV disease were generated. The solid line indicates BAF250a-deficient cases and dotted line indicates BAF250a-retained cases. The 5-year overall survival rate was $16 \%$ in BAF250a-deficient cases and $25 \%$ in BAF250a-retained expression cases $(\mathrm{P}=0.21)$.

chemoresistance of CCA (14); however, the others did not show any significant differences $(10,15-17)$. This discrepancy may be simply explained by a sample size or different patient characteristics. In the present study, BAF250a expression status was not significantly correlated with response rate for chemotherapy of CCA in accordance with the results of several reports $(10,15,16)$.

In the present study, BAF250a-deficient cases exhibited a relatively lower chemotherapy response and a worse prognosis; however, BAF250a expression status was not significantly associated with response rate or prognoses in CCA. Additionally, multivariate analyses revealed that BAF250a status was not an independent prognostic factor for progression-free survival and overall survival. More important factors, including age, FIGO stage and residual tumor diameter, were identified as prognostic factors for overall survival in CCAs.

In conclusion, BAF250a expression status was not identified as an independent prognostic factor for progression-free 
峁|

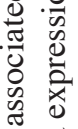

至

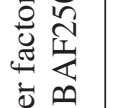

吾愛

告

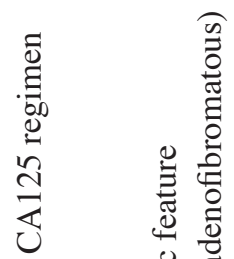

i $\frac{0^{\circ}}{\pi}$

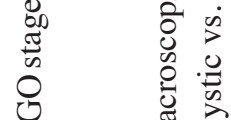

苮
0
0
0
0
0
0
0
0
0
0



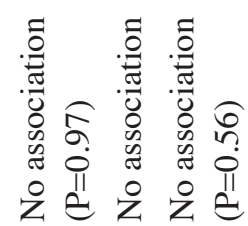

$\frac{2}{2}$

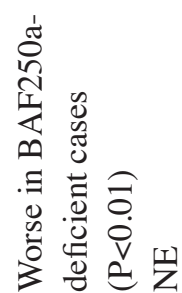

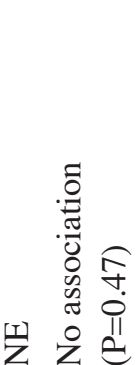

2

ป̊ จे

ஃ̊ ถீ

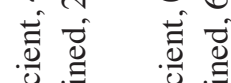

过

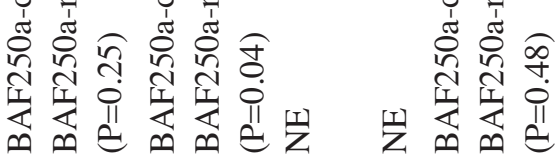

旅

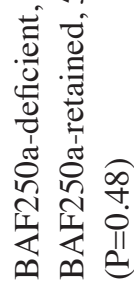

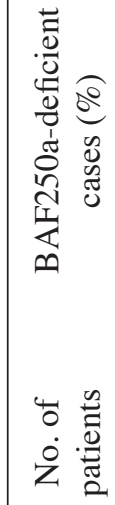

(1)

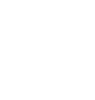

ले $\because$ in 78 
survival or overall survival in patients with CCA. However, BAF250a-deficient expression was closely correlated with early tumorigenesis of endometriosis-related CCA. BAF250a expression was closely associated with an early neoplastic process of endometriosis and it may be a potential biomarker for detecting early malignant transformation of endometriosis. Additionally, it is necessary to investigate the method to detect the alteration of BAF250a expression in the follow-up of the patients with endometriosis. Further biomarker analyses, including BAF250a expression, are required to improve the prognoses of CCAs.

\section{References}

1. Chan JK, Teoh D, Hu JM, Shin JY, Osann K and Kapp DS: Do clear cell ovarian carcinomas have poorer prognosis compared to other epithelial cell types? A study of 1411 clear cell ovarian cancers. Gynecol Oncol 109: 370-376, 2008.

2. Sugiyama T, Kamura T, Kigawa J, Terakawa N, Kikuchi Y, Kita T, Suzuki M, Sato I and Taguchi K: Clinical characteristics of clear cell carcinoma of the ovary: A distinct histologic type with poor prognosis and resistance to platinum-based chemotherapy. Cancer 88: 2584-2589, 2000.

3. Itamochi H, Kigawa J and Terakawa N: Mechanisms of chemoresistance and poor prognosis in ovarian clear cell carcinoma Cancer Sci 99: 653-658, 2008.

4. Takano M, Kikuchi Y, Yaegashi N, Kuzuya K, Ueki M, Tsuda H, Suzuki M, Kigawa J, Takeuchi S, Tsuda H, et al: Clear cell carcinoma of the ovary: A retrospective muticentre experience of 254 patients with complete surgical staging. Br J Cancer 94: 1369-1374, 2006.

5. Gross AL, Kurman RJ, Vang R, Shih IeM and Visvanathan K: Precursor lesions of high-grade serous ovarian carcinoma: Morphological and molecular characteristics. J Oncol 2010: $126295,2010$.

6. Kurman RJ and Shih IeM: The origin and pathogenesis of epithelial ovarian cancer: A proposed unifying theory. Am J Surg Pathol 34: 433-443, 2010.

7. Tan DS and Kaye S: Ovarian clear cell adenocarcinoma: A continuing enigma. J Clin Pathol 60: 355-360, 2007.

8. Wiegand KC, Shah SP, Al-Agha OM, Zhao Y, Tse K, Zeng T, Senz J, McConechy MK, Anglesio MS, Kalloger SE, et al: ARID1A mutations in endometriosis-assciated ovarian carcinoma. N Engl J Med 363: 1532-1543, 2010.

9. Jones S, Wang TL, Shih IeM, Mao TL, Nakayama K, Roden R, Glas R, Slamon D, Diaz LA Jr, Vogelstein B, et al: Frequent mutations of chromatin remodeling gene ARID1A in ovarian clear cell carcinoma. Science 330: 228-231, 2010.
10. Yamamoto S, Tsuda H, Takano M, Tamai S and Matsubara O: PIK3CA mutaitons and loss of ARID1A protein expression are early events in the development of cystic ovarian clear cell adenocarcinoma. Virchows Arch 460: 77-87, 2012.

11. Tan DS, Iravani M, McCluggage WG, Lambros MB, Milanezi F, Mackay A, Gourley C, Geyer FC, Vatcheva R, Millar J, et al: Genomic analysis reveals the molecular heterogeneity of ovarian clear cell carcinomas. Clin Cancer Res 17: 1521-1534, 2011.

12. Yamamoto S, Tsuda H, Yoshikawa T, Kudoh K, Kita T, Furuya K, Tamai S and Matsubara O: Clear cell adenocarcinoma associated with clear cell adenofibromatous components: A subgroup of ovarian clear cell adenocarcinoma with distinct clinicopathologic characteristics. Am J Surg Pathol 31: 999-1006, 2007.

13. Veras E, Mao TL, Ayhan A, Ueda S, Lai H, Hayran M, Shih IeM and Kurman RJ: Cystic and adenofibromatous clear cell carcinomas of the ovary: Distinctive tumors that differ in their pathogenesis and behavior: A clinicopathologic analysis of 122 cases. Am J Surg Pathol 33: 844-853, 2009.

14. Katagiri A, Nakayama K, Rahman MT, Rahman M, Katagiri H, Nakayama N, Ishikawa M, Ishibashi T, Iida K, Kobayashi $\mathrm{H}$, et al: Loss of ARID1A expression is related to shorter progression-free survival and chemoresistance in ovarian clear cell carcinoma. Mod Pathol 25: 282-288, 2012.

15. Yokoyama Y, Matsushita Y, Shigeto T, Futagami M and Mizunuma H: Decreased ARID1A is correlated with chemoresistance in epithelial ovarian cancer. J Gynecol Oncol 25: 58-63, 2014.

16. Maeda D, Mao TL, Fukuyamaa M, Nakagawa S, Yano T, Taketani Y and Shih IeM: Clinicopathological significance of loss of ARID1A immunoreactivity in ovarian clear cell carcinoma. Int J Mol Sci 11: 5120-5128, 2010.

17. Lowery WJ, Schildkraut JM, Akushevich L, Bentley R, Marks JR, Huntsman D and Berchuck A: Loss of ARID1 A-associated protein expression is a frequent event in clear cell and endometrioid ovarian cancers. Int J Gynecol Cancer 22: 9-14, 2012.

18. Kato M, Yamamoto S, Takano M, Matsubara O and Furuya K: Aberrant expression of mammalian target of rapamycin, hypoxia-inducible factor- $1 \alpha$ and glucose transporter 1 in the development of ovarian clear-cell adenocarcinoma. Int J Gynecol Pathol 31: 254-263, 2012.

19. Samartzis EP,Samartzis N,Noske A,FedierA,CaduffR,Dedes KJ, Fink D and Imesch P: Loss of ARID1A/BAF250a-expression in endometriosis: A biomarker for risk of carcinogenic transformation? Mod Pathol 25: 885-892, 2012.

20. Xiao W, Awadallah A and Xin W: Loss of ARID1A/BAF250a expression in ovarian endometriosis and clear cell carcinoma. Int J Clin Exp Pathol 5: 642-650, 2012.

21. Ayhan A, Mao TL, Seckin T, Wu CH, Guan B, Ogawa H, Futagami M, Mizukami H, Yokoyama Y, Kurman RJ and Shih IeM: Loss of ARID1A expression is an early molecular event in tumor progression from ovarian endometriotic cyst to clear cell and endometrioid carcinoma. Int J Gynecol Cancer 22: $1310-1315,2012$ 